\title{
Place de l'anglais et paradoxes des apprentissages langagiers à l'école
}

\section{Gilles Forlot}

\section{(2) OpenEdition}

\section{Journals}

Édition électronique

URL : https://journals.openedition.org/rdlc/2013

DOI : $10.4000 /$ rdlc. 2013

ISSN : 1958-5772

Éditeur

ACEDLE

Référence électronique

Gilles Forlot, "Place de l'anglais et paradoxes des apprentissages langagiers à l'école », Recherches en didactique des langues et des cultures [En ligne], 7-1 | 2010, mis en ligne le 01 avril 2010, consulté le 12 avril 2022. URL : http://journals.openedition.org/rdlc/2013 ; DOI : https://doi.org/10.4000/rdlc.2013

Ce document a été généré automatiquement le 12 avril 2022

\section{(9) $\odot \Theta \Theta$}

Recherches en didactique des langues et des cultures is licensed under a Creative Commons AttributionNonCommercial-NoDerivatives 4.0 International License 


\title{
Place de l'anglais et paradoxes des apprentissages langagiers à l'école
}

\author{
Gilles Forlot
}

\section{Introduction}

1 Partons d'une évidence difficilement contestable : non seulement l'anglais domine très largement comme langue étrangère apprise dans les systèmes scolaires européens, mais il est en nette croissance à mesure que les années passent (cf. statistiques plus bas). Aussi, si l'on prend le contexte de la France, le paradoxe de la mise en place des langues au primaire a peut-être été que cet enseignement a renforcé la position dominante de l'anglais en faisant descendre la "demande sociale" (je reviens à ce concept plus loin) d'un cran : de la $6^{e}$, première classe de l'enseignement secondaire, on est passé au cycle des approfondissements de l'école primaire, dit cycle III (CE2, CM1, $\mathrm{CM} 2$ ), puis récemment au cycle des apprentissages fondamentaux (dit cycle II, à savoir dès l'âge de 6 ans). On peut donc penser que dans quelques années, le cycle II marquera le début de l'anglo-centrisme. Ce terme n'a pas ici forcément de connotation péjorative, mais il fait écho au titre de cet article : l'anglais est à l'hyper-centre des apprentissages linguistiques dans le système scolaire français. Face à cet état de fait, comment peut-on penser qu'un véritable plurilinguisme puisse un jour se développer au sein (et au-delà) de notre système scolaire ? Je commencerai par faire un survol de quelques données historiques et statistiques, puis j'exposerai quelques paradoxes qui me semblent, parce qu'ils sont au cœur de l'enseignement des langues en France, agir comme des obstacles au développement d'une "philosophie" plurilingue dans le système scolaire. 


\section{Quelques jalons historico-linguistiques européens}

\subsection{Le passage du français à l'anglais en Europe}

2 Selon C.Truchot (2008), le français a su résister durant les quarante premières années de la CEE parce qu'il s'agissait de la langue de trois des pays fondateurs (Belgique, Luxembourg, France) et parce que le nombre de fonctionnaires francophones dans l'administration européenne permettait une veille francophone, de surcroît activement soutenue par les fonctionnaires français eux-mêmes. Il n'est pourtant pas sûr que l'adhésion à la Communauté européenne du Royaume-Uni et de la République d'Irlande en 1973 ait eu un impact expansionniste très notoire. En fait, on peut penser que la diffusion de l'anglais coïncide non seulement avec l'adhésion des deux pays à la CEE, mais aussi au contexte des années 1970 durant lesquelles la langue anglaise se "véhicularise" en Europe et dans le monde. Truchot (2008) souligne qu'en dehors de tout cadre juridique explicite, l'anglais est devenue à bien des égards la seule langue de communication semi-interne et externe entre les composantes et les partenaires des institutions européennes (cf. aussi Labrie, 1993) ${ }^{1}$. Tout ceci nous mène à la question de l'enseignement des langues dans l'Europe communautaire, non seulement à un échelon supranational mais aussi à un niveau plus local, car désormais, des directives européennes existent.

\subsection{La question éducative dans les institutions européennes}

3 Dans le domaine des langues, si l'on excepte les règlements communautaires concernant la production et la traduction des textes et directives légales, la capacité que s'est donnée l'Union Européenne (U.E.) est celle de l'orientation éducative en matière linguistique. L'une des dates clés est la création en 1987 des programmes Erasmus. Le traité de Maastricht (1992) autorise les Institutions européennes à soutenir les états dans leurs politiques de mobilité pouvant avoir un impact sur l'apprentissage ou le perfectionnement linguistique (même si aucun objectif clair n'est ainsi fixé). On recense par exemple le programme Socrates, comprenant les sous-programmes Comenius, pour l'enseignement scolaire, et Erasmus pour l'enseignement supérieur, le programme Grundtvig destiné à la formation des adultes, ainsi que le programme Leonardo da Vinci, consacré à la formation professionnelle, qui inclut depuis 1995 un programme de promotion du plurilinguisme et de formation linguistique des enseignants appelé Lingua.

On pourra regretter, à l'instar de Claude Truchot, que l'on ne se soit pas assez intéressé à l'impact linguistique de ces programmes éducatifs européens. L'intuition et le sens commun peuvent nous orienter dans diverses directions et ainsi nous induire en erreur; a priori, le nombre, la diversité et la promotion des langues de l'U.E. (27 langues officielles en 2007), voire l'équilibre démographique pour les langues majeures de l'Union (anglais, allemand, français, italien et dans une moindre mesure l'espagnol) laisseraient penser que l'Europe est en train de se constituer une culture véritablement plurilingue et diversifiée... Mais il n'en est rien et bizarrement, cela n'étonnera personne : entre 1990 et 1994, 50 \% des séjours de formation (si l'on se réfère au total des programmes européens ci-dessus) ont eu lieu dans un pays anglophone (Hermans, 1997, cité par Truchot, 2008 : 91). 
On notera que le cabinet de consultants Deloitte et Touche a réalisé une évaluation des résultats des programmes Socrates et Leonardo en 2003. Le rapport d'évaluation indique que malgré de gros moyens financiers mis en œuvre, l'impact des programmes a essentiellement bénéficié aux langues déjà connues et que peu de stagiaires ont entrepris d'apprendre une ou des langues nouvelles ${ }^{2}$. On peut en percevoir les raisons dans l'autre observation du rapport d'évaluation : la Commission et les partenaires ont géré, durant les périodes évaluées (et gageons que cela n'a guère changé), les projets en anglais à $80 \%$, alors qu'à peine $13 \%$ de ces projets provenaient de pays anglophones (Royaume-Uni, Irlande). Cette pratique paraîtra paradoxale dans la mesure où ces projets visaient au développement de compétences plurilingues et de diversification linguistique. Or, postuler à ces projets requiert la maîtrise de l'anglais, ce qui acte la reproduction de son statut de langue incontournable. Pour Truchot,

la langue anglaise semble bien être devenue un élément identificatoire du programme Erasmus; dans la mesure où celui-ci peut générer un sentiment d'appartenance à l'Europe, la langue anglaise deviendrait donc son expression privilégiée (Truchot, 2008 : 94).

6 Avant de nous tourner vers l'examen de la situation de l'enseignement-apprentissage anglo-centrique en France, ajoutons que cet état de fait européen est à la fois une conséquence de l'expansion de la langue anglaise en Europe et dans le monde, mais qu'il y contribue aussi largement. Dit en des termes sociologiques, l'anglais produit et reproduit de la domination, de l'hégémonie, de l'inégalité entre les langues en présence, créant ainsi les conditions de sa propre diffusion et de sa domination. On ne peut plus se passer de lui, même dans les instances (cf. plus haut) où on vise justement à en limiter les effets. On assiste à l'émergence d'une situation sociolinguistique "hypermoderne" liée à la mondialisation (cf. entre autres Fairclough, 2006 ; Heller, 2007), situation dans laquelle le plurilinguisme ne peut se construire que sur les bases d'une dualité entre anglais et une autre langue. Cette dualité est la condition nécessaire à la survie, précisément, de l'autre langue. Le principe, en somme, se résumerait à cette injonction : "vivons avec l'anglais pour ne pas disparaître...".

\section{L'anglais dans le système éducatif français}

\section{1. État des lieux de l'apprentissage des langues et de l'anglais}

Inutile d'y consacrer de longs paragraphes. Tout le monde le sait, l'anglais domine très largement à tous les échelons du système éducatif français, et ce bien avant que le rapport de Jacques Attali, remis en 2008 au Président de la République, n'explique que pour libérer la croissance, il y a lieu de "se donner les moyens que tout élève maîtrise avant la fin de la $6^{e}$ le français, la lecture, l'écriture, le calcul, le travail de groupe, l'anglais et l'informatique" (Attali, 2008: 14) et de "repenser le socle commun des connaissances pour y ajouter le travail en groupe, l'anglais, l'informatique et l'économie" (Attali, 2008: 26). Ces extraits font écho aux déclarations récentes (2008) du ministre français de l'éducation nationale, Xavier Darcos, sur les problèmes posés par la maîtrise insuffisante de l'anglais par les jeunes diplômés qui sortent du lycée. D'ores et déjà, la question de la conversion de l'anglais langue centripète de facto à un statut de langue obligatoire de jure se pose... 
8 Les deux tableaux ci-dessous le montrent bien: l'anglais est devenu largement hégémonique et si la France n'a pas communiqué ses résultats pour ce qui concerne l'enseignement primaire, cela est peut-être dû à la lente généralisation de cet enseignement en cycle 2. Si l'on consulte le document Repères et références statistiques sur les enseignements, la formation et la recherche-dit RERS-de 2007, on peut pourtant relever certains chiffres concernant la répartition en pourcentage de l'enseignement des LVE au cycle III de l'école primaire.

TABLEAU 1 - Apprentissage des langues au cycle III (CE2-CM1-CM2) de l'école primaire en France

\begin{tabular}{|l|l|l|l|}
\hline $\begin{array}{l}\text { Pourcentage de groupes d'élèves... } \\
\text { / à la rentrée }\end{array}$ & $\begin{array}{l}\text {...qui étudient } \\
\text { l'ALLEMAND }\end{array}$ & $\begin{array}{l}\text {...qui étudient } \\
\text { l'ANGLAIS }\end{array}$ & $\begin{array}{l}\text {...qui étudient } \\
\text { l'ESPAGNOL }\end{array}$ \\
\hline 2001 & 18,6 & 76,4 & 02,0 \\
\hline 2002 & 16,4 & 78,6 & 02,2 \\
\hline 2003 & 15,1 & 79,6 & 02,4 \\
\hline 2004 & 11,5 & 81,6 & 02,0 \\
\hline 2005 & 13,1 & 82,6 & 02,3 \\
\hline 2006 & 10,6 & 86,0 & 01,8 \\
\hline
\end{tabular}

Tableaux 2a - Apprentissages des langues dans l'enseignement secondaire en France

\begin{tabular}{|l|l|l|}
\hline 2a) En LV1 & anglais & $93 \%$ \\
\hline & allemand & $8 \%$ \\
\hline & Autres langues & $2 \%$ \\
\hline $\begin{array}{l}\text { Notes: (a) En France, } 5 \text { millions d'enfants scolarisés dans le secondaire étudient l'anglais en } \\
\text { première langue; (b) l'addition des pourcentages ci-dessus dépassent 100\% car les sections } \\
\text { "bilangues" (2 LV1) se développent : elles concernaient 5\% des collégiens en 2004 et } 9 \% \text { de ceux-ci } \\
\text { en 2006. }\end{array}$ \\
\hline
\end{tabular}

Tableaux $2 b$ - Apprentissages des langues dans l'enseignement secondaire en France - suite

\begin{tabular}{|l|l|l|}
\hline 2b) En LV2 & allemand & $16 \%$ au lycée, 13 \% au collège \\
\hline & espagnol & $71 \%$ dans le secondaire \\
\hline Note : 99000 élèves du secondaire (soit $6,5 \%$ ) étudient une LV3 \\
\hline
\end{tabular}


Tableaux 2c - Apprentissages des langues dans l'enseignement secondaire en France - suite/fin

\begin{tabular}{|l|l|}
\hline 2c) Bilan pour l'enseignement secondaire & l'anglais \\
\hline $97 \%$ des élèves du secondaire en France étudient... & l'espagnol \\
\hline $40 \%$ des élèves du secondaire en France étudient... & l'allemand \\
\hline $15 \%$ des élèves du secondaire en France étudient... & l'italien \\
\hline $4 \%$ des élèves du secondaire en France étudient... & sauf l'allemand \\
\hline De 2000 à 2006, toutes les langues ont connu des augmentations d'effectifs... & \\
\hline
\end{tabular}

\subsection{L'enseignement des langues, un art du parallélisme et de la juxtaposition}

\subsubsection{Le problème de la langue}

9 L'école primaire est le haut lieu de l'apprentissage langagier formel et l'instauration de l'enseignement d'une langue procède de cette vision, partagée par le sens commun ${ }^{3}$ que la LVE va se construire efficacement dès le primaire pour se poursuivre au secondaire. Ainsi, la présence de deux ou plusieurs langues, à savoir d'une part le français langue de scolarisation et d'autre part une ou plusieurs langues étrangères en situation bien évidemment de déséquilibre patent - la LVE ne constituant que $4 \%$ du temps total d'enseignement - allait peut-être, dans l'esprit de certains (du sens commun en tous cas ; cf. Forlot, 2006 et 2009a), participer de l'amélioration sensible des compétences des jeunes Français en fin de scolarité secondaire générale. Rappelons tout de même que les programmes français, inspirés du Cadre européen de référence pour les langues (Conseil de l'Europe, 2001), préconisent un niveau en fin de terminale (langue vivante 1) se situant autour de B2, c'est-à-dire celui du locuteur indépendant.

\subsubsection{Le problème de la culture/civilisation}

10 Abordons désormais les choses différemment, par un biais culturel. L'un des objectifs primordiaux de l'école primaire est la découverte du monde qui nous entoure et l'apprentissage d'une langue nouvelle, dans le cadre limitatif qui est le sien (entre 60 et 90 minutes d'apprentissage par semaine), consiste aussi en la découverte d'une culture autre, celle de nos voisins européens, par exemple. En fait, la généralisation de l'anglais a effectivement permis de créer les conditions d'un apprentissage nouveau, à la fois linguistique et culturel. Mais le premier volet paraît fortement centré sur la dimension lexicale et la répétition (cf. Chini, 2009, à par.) et le second volet semble par trop stéréotypé et folklorisant: le petit déjeuner anglais, Halloween, les Christmas crackers, etc. Quid alors, précisément pour l'anglais d'ailleurs, de la complexité et de la diversité des sociétés anglo-saxonnes, tant d'un point de vue externe qu'interne? En effet, ces sociétés présentent des différentes notoires entre elles et, partant, il devient illégitime de se focaliser sur la seule Grande-Bretagne; d'autre part, on oublie trop souvent que 
ce sont souvent des sociétés multiculturelles, "hybridées" (cf. Jucquois, 2003) et diverses ${ }^{4}$.

\subsubsection{Approfondissement : de quelques pratiques pédagogiques actuelles}

11 Examinons avec davantage d'attention quelques pratiques courantes auxquelles tous les formateurs sont confrontés - ils y contribuent parfois, du reste... - au quotidien dans les modules de formation à l'enseignement des LVE dans le primaire, notamment dans les Instituts universitaires de formation des maitres (IUFM).

On mentionnera d'abord que le premier réflexe, somme toute compréhensible, des professeurs en formation, au primaire comme au secondaire, est la recherche de manuels proposant des activités linguistiques et culturelles "clés en mains", souvent décrochées des situations de communication dont les enfants peuvent ressentir le besoin. Au secondaire, il peut arriver d'entendre des inspecteurs pédagogiques se plaindre que les enseignants ne sachent pas assez s'affranchir de leurs manuels scolaires. Les progrès réalisés par l'enseignement des langues au primaire, où soufflait un vent d'originalité pédagogique il y a une dizaine d'années, se trouvent ralentis par l'émergence de modèles pédagogiques inspirés du secondaire (notamment certaines formes de "prêt-à-enseigner" qu'offrent les manuels scolaires...) $)^{5}$.

13 Ensuite, il existe à mon sens une distinction fréquente, dans les séquences d'enseignement des langues à l'école primaire, entre le culturel/civilisationnel et le linguistique (cf. point 3.2.2.) ${ }^{6}$. Elle crée les conditions d'un dédoublement de l'enseignement des langues au primaire, allant parfois jusqu'à la présence de deux professeurs chargés de l'enseignement de la langue (ou, disons, de l'anglais dans de nombreuses écoles de nos jours). D'un côté, on trouve le "linguiste", qui s'occupe de la langue et organise ses séquences d'enseignement avec des priorités souvent fort lexicalistes (les vêtements, les fruits, les animaux de la ferme, mais aussi quelques structures typiques permettant d'y injecter du lexical : l'expression de la possession, du souhait...) ; de l'autre côté, le "civilisationniste" (qui est parfois le même enseignant...) s'occupe de l'enseignement culturel, dispensant un savoir parfois frontal sur divers aspects de la vie quotidienne ou de l'histoire, sous la forme de séances stéréotypiques (et stéréotypantes) sur, si l'on prend l'exemple de l'anglais, Thanksgiving, Guy Fawkes Day, la livre sterling, la Famille Royale d'Angleterre...

\subsubsection{Quand l'enseignant fut un apprenant...}

14 La construction de ces parallélismes est à n'en pas douter un reflet d'une caractéristique du cloisonnement que connaissent à la fois l'institution LVE en France (la monovalence des enseignants de langues en étant un bel exemple) et l'enseignement sur le terrain de ces LVE (voir Castellotti, à par. 2009; Forlot, à par. 2009b). L'enseignement des langues a suivi un modèle classique, proche du modèle traditionnel latiniste, du collège au lycée ${ }^{7}$. La segmentation des apprentissages à l'issue desquels la mise en relation des éléments, pensait-on, allait produire de la communication a aussi fait en sorte que la méthode communicative, prônée dès la fin des années 1980 dans le système scolaire français, n'a en réalité eu qu'un très modeste impact sur la pédagogie des LVE en France. Le tableau suivant indique comment les professeurs des écoles en formation (appelés PE2) dans deux IUFM, à Amiens et à Strasbourg, perçoivent la façon dont l'enseignement des langues leur a été dispensé. 
Tableau 4 - Perceptions de professeurs des écoles stagiaires : l'enseignement des LVE au collège et au lycée

\begin{tabular}{|c|c|c|c|}
\hline $\begin{array}{l}\text { Perception de l'enseignement } \\
\text { des LVE, collège et lycée (2008) }\end{array}$ & & $\begin{array}{l}\text { Réponses PE2 } \\
\text { IUFM Picardie } \\
(\mathrm{N}=71)\end{array}$ & $\begin{array}{l}\text { Réponses PE2 } \\
\text { IUFM Alsace } \\
(\mathrm{N}=54)\end{array}$ \\
\hline \multirow[t]{5}{*}{$\begin{array}{l}\text { Au collège, l'enseignement des } \\
\text { LV a paru centré }\end{array}$} & $\begin{array}{l}\text { A) sur la communication } \\
\text { et l'interaction }\end{array}$ & $25,3 \%$ & $35,1 \%$ \\
\hline & B) sur le grammatical & $83,0 \%$ & $81,5 \%$ \\
\hline & C) sur le lexical & $69,0 \%$ & $68,5 \%$ \\
\hline & $\begin{array}{l}\text { D) sur la civilisation/la } \\
\text { culture }\end{array}$ & $14,0 \%$ & $20,4 \%$ \\
\hline & E) sur la traduction & $14,1 \%$ & $18,5 \%$ \\
\hline \multirow[t]{5}{*}{$\begin{array}{l}\text { Au lycée, l'enseignement des LV } \\
\text { a paru centré }\end{array}$} & $\begin{array}{l}\text { A) sur la communication } \\
\text { et l'interaction }\end{array}$ & $28,2 \%$ & $35,1 \%$ \\
\hline & B) sur le grammatical & $67,6 \%$ & $70,4 \%$ \\
\hline & C) sur le lexical & $64,7 \%$ & $57,4 \%$ \\
\hline & $\begin{array}{l}\text { D) sur la civilisation/la } \\
\text { culture }\end{array}$ & $35,2 \%$ & $38,9 \%$ \\
\hline & E) sur la traduction & $39,4 \%$ & $22,2 \%$ \\
\hline
\end{tabular}

Source : Forlot, en préparation

On constate, dans ces deux tableaux, la domination d'approches perçues comme grammaticales et lexicales ${ }^{8}$. Cela laisse entendre que les modèles classiques perdurent jusqu'à ce que les professeurs arrivent en stage sur le terrain. Les formations universitaires et les concours de recrutement portent eux aussi une part importante de responsabilités, parce qu'ils produisent et reproduisent (mais peut-être est-ce leur rôle ?) ce que l'enseignant ne devra précisément plus faire devant ses élèves : du magistral, du frontal, de l'hypernormatif et, sur le plan des contenus, du civilisationnel détaché du culturel général (le littéraire notamment), du linguistique (générativiste ou, plus souvent, "énonciativiste") détaché de pratiques langagières "réelles"'.

Tableau 5 - Perceptions de professeurs des écoles stagiaires : l'enseignement des LVE à l'université

\begin{tabular}{|l|l|l|l|}
\hline $\begin{array}{l}\text { Perception de } \\
\text { l'enseignement des LVE, } \\
\text { université (2008) }\end{array}$ & $\begin{array}{l}\text { Réponses PE2 } \\
\text { IUFM Picardie } \\
(\mathrm{N}=71)\end{array}$ & $\begin{array}{l}\text { Réponses PE2 } \\
\text { IUFM Alsace } \\
(\mathrm{N}=54)\end{array}$ \\
\hline
\end{tabular}




\begin{tabular}{|l|l|l|l|}
\hline $\begin{array}{l}\text { À l'université, } \\
\text { l'enseignement des LV a } \\
\text { paru centré }\end{array}$ & $\begin{array}{l}\text { A) sur la communication et } \\
\text { l'interaction }\end{array}$ & $32,4 \%$ & $40,7 \%$ \\
\hline & B) sur le grammatical & $26,8 \%$ & $11,1 \%$ \\
\hline & C) sur le lexical & $31,0 \%$ & $29,6 \%$ \\
\hline & D) sur la civilisation/la culture & $38,0 \%$ & $53,7 \%$ \\
\hline & E) sur la traduction auto- & $43,7 \%$ & $35,2 \%$ \\
\hline & $\begin{array}{l}\text { Enseignement en aprentissage, } \\
\text { comme 'non-enseignement' }\end{array}$ & s/o & $7,4 \%$ \\
\hline
\end{tabular}

Source : Forlot, en préparation

16 On ajoutera que les concours - agrégations, $\mathrm{CAPES}^{10}, \mathrm{CAPLP} .$. - dans leur format actuel, ne contribuent que très peu à développer les approches plurielles des langues, l'ouverture didactique au plurilinguisme et, sur un plan tout bonnement pédagogique, la pratique de l'enseignement à des publics devenus pluriculturels et très certainement ouverts à des pistes de travail interlinguistique. Je ne développerai ici ni la forte sélection sociale que ces concours imposent au milieu éducatif - il faut relire Bourdieu (1984:126-128) et l'anecdote de l'agrégé béarnais - ni la nette tendance à la création d'une "marchandification"11 des langues dans le milieu universitaire français, où pléthores de formations et de publications tournent autour des programmes des concours de recrutement. Ces réflexions concernent bien sûr au premier chef l'anglais.

\subsection{Pressions de la norme et enseignements-apprentissages paradoxaux}

17 Je laissais donc entendre que l'enseignement scolaire des langues en France est encore soumis à des schémas inspirés d'approches plutôt "classiques" (enseignements de la grammaire, de la littérature, de la civilisation), ce qui apparaît tout à fait paradoxal pour la langue de la communication internationale globalisée, l'anglais, devenu, qu'on le veuille ou non, l'idiome des flots transculturels et des traits culturels et langagiers globaux réappropriés localement (voir Pennycook, 2007212). Concernant la norme et le développement du prétendu "Globish" dont tout le monde parle sans en avoir défini les contours et sans en avoir démontré l'existence de façon convaincante (ibid. ; Makoni \& Pennycook, 2007), on pourra ironiquement renouveler notre confiance aux anglicistes, qui veillent à l'interdiction des split infinitives (l'insertion d'un morphème entre to et "sa" base verbale. Ex. : to quickly go), des if hypothétiques suivis de l'auxiliaire would (en France, on dit que "les si n'aiment pas les '-rai-'..."), des like I + verbe (ex. : like I said au lieu de as I said), pourtant si courants dans le monde anglo-saxon, notamment aux États-Unis. Il ne suffit pas en effet qu'une majorité de locuteurs anglophones utilisent ces formes pour que s'atténuent les réflexes épilinguistiques les plus fréquents chez les enseignants français : la sacralisation, la fétichisation et la totémisation de la langue 
(Bourdieu, 1982 ; Kramsch, 1998; Klinkenberg, 2001), ainsi qu'une approche souvent puriste de celle-ci (Paveau et Rosier, 2008).

18 En termes plus pédagogiques, on serait en droit de questionner la pertinence de l'apprentissage des langues au primaire si on le lexicalise à outrance et si, en somme, les enjeux pour les élèves sont si minimes, tant on juxtapose les apprentissages (de façon anti-spiralaire, pour ainsi dire) comme on le fait encore de nos jours. L'exposition des enfants aux LVE à l'école primaire est certainement très bénéfique, mais comment ne pas se demander si les circonstances actuelles dans lesquels cet enseignement se déroule ne desservent pas sa cause : des enseignants peu formés (en langue comme en didactique), une compartimentation des disciplines, une tendance au retour de pratiques traditionnelles sans doute liées aux nouvelles priorités du Ministère de l'éducation nationale en termes de "socle commun". Ces priorités ont entraîné un retour de l'écrit et de l'apprentissage par cœur et une mise à l'index des principes de l'observation réfléchie de la langue au primaire .... ${ }^{13}$.

Il apparaît que la génération actuelle de professeurs des écoles, soumise à la pression de l'enseignement des langues à l'école, est confrontée à deux séries de situations paradoxales :

1. Une demande de diversité linguistique à l'école qui permettrait à toutes les compétences de s'affirmer et d'être mises à profit vs une forte demande d'anglais.

2. Une situation inconfortable, vis-à-vis des autres acteurs de l'école (parents, inspecteurs, collègues...) où les enseignants sont à la fois prestataires et consommateurs (potentiels) d'enseignement-apprentissage des langues et surtout d'anglais.

Le système éducatif (et par là même la plupart de ses acteurs, qu'ils soient enseignants, inspecteurs, parents...) est sans doute passablement schizolingue (cf. Forlot, 2009a), obéissant tant bien que mal à des injonctions paradoxales (voir aussi Castellotti, 2008b) : développer le plurilinguisme et l'ouverture à la pluralité dans l'espace scolaire tout en répondant implicitement à la demande sociale très forte du "tout anglais" et "du plus en plus d'espagnol". Le paradoxe est aussi d'encourager la diversité, la pluralité et la variété des pratiques langagières (par les pratiques artistiques et musicales telles le rap, le slam, etc.) tout en se conformant bon gré mal gré aux injonctions de renforcer la concentration sur la norme, sur l'écrit, sur les conjugaisons, sur la préséance symbolique de la "langue nationale"...

\subsection{Hors de l'école : l'anglais, les parents et la "demande sociale"}

21 La "demande sociale", dans le domaine linguistique, peut s'interpréter comme la combinaison de la compréhension, par les acteurs sociaux d'un espace donné, du marché linguistique actuel et de leurs représentations sur les langues. Il s'agirait entre autres des points suivants.

\subsubsection{La soumission à des pressions de nature épilinguistique ${ }^{14}$}

Ces pressions sont de différentes natures : d'une part, on s'intéresse à la position du français dans le monde, à la nécessité de le promouvoir à l'extérieur de nos frontières et dans l'Hexagone lui-même. On peut renvoyer ici aux discours sur lesquels s'appuient les positionnements des rapports Benisti (en 2005) et Bentolila (2006) et leurs effets sur 
la politique linguistique implicite dans le système scolaire français, déjà abordés au point 3.3 ci-dessus.

En second lieu, on peut s'intéresser à la position des langues en France, liée notamment à leurs supposées vertus utilitaires. Dans ce domaine, l'anglais triomphe et le chinois prend son envol, alors que l'italien ou le portugais n'ont jamais vraiment eu de poids, n'étant pas porteurs de ces supposées vertus.

En dernier lieu, on mentionnera que la situation des langues à l'international exerce bien sûr une pression constante, et là aussi, l'anglais ne peut évidemment que bénéficier de son rôle de prétendue ${ }^{15}$ lingua franca.

\subsubsection{La soumission à des pressions de nature métalinguistique}

Il s'agit ici des classiques du genre en termes de représentations et de stéréotypes sociolinguistiques: l'anglais et l'espagnol langues faciles, alors que le français est la langue "la plus difficile du monde" (sic) ; l'allemand est "une langue laide et difficile", etc. Je renvoie ici à moult publications éclairantes sur le sujet : Zarate, 1993; Matthey, 1997, Castellotti, 2001; Alén Garabato et al., 2003; Moore, 2007 ; pour un terrain spécifique aux enseignants du primaire, voir Forlot 2006 et 2009a).

Aussi, il est indéniable que l'anglais profite, dans le système scolaire français, de cette double pression issue de la "demande sociale" : langue internationale, indispensable et perçue somme toute comme assez facile. À l'école, la "demande sociale" est issue, pour $l^{\prime}$ essentiel, des parents ${ }^{16}$, dont les enfants relayent les discours circulant ${ }^{17}$ dans l'espace public: importance de parler anglais de nos jours, facilité de l'apprendre dès le plus jeune âge, accès à pléthores de ressources commerciales (dont certaines en ligne).

Il est probable que les nouvelles orientations ministérielles, qui entendent associer davantage les parents aux programmes de l'école primaire, ira dans le sens d'un renforcement de l'anglais. Le travail en interaction de tous les acteurs de l'espace scolaire est indéniablement positif. Les parents sont en effet en demande de connaissances sur ce que leurs enfants apprennent, et dans le domaine des langues, ils sont fortement demandeurs d'anglais. Néanmoins, il serait sans doute utile de participer à leur "éducation" sociolinguistique, c'est-à-dire les amener à comprendre l'importance de la diversité linguistique, de l'approche transdisciplinaire des langues, des démarches orales (d'inspirations communicative et actionnelle), l'apport fondamental des phases de conceptualisation (la plupart du temps en L1), la place de l'écrit et son articulation avec l'apprentissage de l'oral, etc. Bref, il s'agit de les tenir informés des évolutions de la didactique des langues (se basant notamment sur le CECRL) et de les sensibiliser à une philosophie plurilingue dans l'apprentissage des langues (Young, 2008b). Sans cette démarche de sensibilisation, l'institution scolaire crée les conditions d'une méfiance des parents à l'égard de ses compétences pédagogiques, et fait donc le lit des formations privées, où l'anglais là aussi triomphe. 


\section{L'anglais et le plurilinguisme en situation d'apprentissage : quelles compatibilités?}

\subsection{Les scénarios et leurs effets}

Dans une optique de politique linguistique virtuelle, on pourrait envisager plusieurs scénarios relatifs à l'enseignement des langues dans l'institution scolaire.

\subsubsection{Un apprentissage où l'anglais serait interdit}

Ce scénario est celui des pays où l'anglais a-ou avait-une forte connotation idéologique inverse à celui du régime en place (la Corée du Nord, l'Union Soviétique et ses satellites...). De telles pratiques paraissent contre-productives, car elles renforcent à la fois les représentations négatives des langues (l'anglais en l'occurrence), parce qu'elles créent un vide que d'autres, dans une économie libéralisée, viendront irrémédiablement combler par la création de cours particuliers, d'écoles de langues privées, etc., produits du marché linguistique concurrentiel où précisément aucune approche pédagogique autre que celle de l'efficacité n'est prônée.

Si l'on raisonne sur la base de ce scénario, on peut imaginer que le manque créera non seulement l'envie et surtout le besoin d'apprendre, ainsi que la méfiance vis-à-vis d'autorités institutionnelles allant à l'encontre de la "demande sociale". À l'extrême, il est même possible que l'absence de l'anglais renforce ultérieurement son statut: on pense ici notamment à la conversion des anciens pays du bloc soviétique à l'économie de marché, qui a marqué l'abandon massif de la langue russe au profit de la langue anglaise et par la formation "express" d'anciens enseignants de la première à la deuxième ! (voir par exemple Prendergast, 2008, pour la situation slovaque).

\subsubsection{Un apprentissage où l'anglais serait obligatoire}

31 C'est la pratique dans certains états de l'U.E. Cette option est défendue par certaines personnalités plus ou moins engagées en politique (l'économiste A. Minc ou le physicien $\mathrm{C}$. Allègre, par exemple). Néanmoins, elle semble à bon nombre de citoyens francophones gênante du fait de la hiérarchisation implicite qu'elle créerait entre les langues de l'école par le biais de l'étiquette obligatoire qui serait accolée à l'anglais. Pratiquement, elle n'est pas absurde dans la mesure où (a) elle ressemble ni plus ni moins à de nombreux cas actuels où l'anglais n'est pas obligatoire : rappelons-nous les $97 \%$ de la population scolaire française qui apprennent l'anglais actuellement... ; (b) elle pourrait et devrait, selon les accords européens, être accompagnée de l'enseignement-apprentissage d'au moins une autre langue.

On pourrait donc envisager un scénario où l'anglais serait obligatoire, revêtant ainsi la fonction de discipline linguistique fondamentale et cédant ainsi la place à un enseignement diversifié de deux autres LVE et, dans l'idéal, des fonctions de langue passerelle aidant à la construction de nouvelles compétences linguistiques (cf. cidessous). 


\subsubsection{Un apprentissage où l'anglais ferait partie des 2 ou 3 langues enseignées}

33 Ce scénario correspond au fonctionnement actuel en France et de nombreux autres pays de l'Union européenne. Le défaut de cette option est qu'il s'agit en fait d'une obligation masquée à l'apprentissage de l'anglais et d'une autre langue ; dans certaines filières technologiques du reste, le choix est bien souvent limité à l'anglais, alors que certaines compétences linguistiques, potentiellement utiles à une coopération professionnelle, se trouvent mises en sommeil chez de nombreux élèves issus des migrations (arabe, portugais pour ne citer que les langues de plus grande diffusion).

cend lieu, certains systemes scolaires ne fonctionnent pas sur le mode de l'apprentissage simultané, mais plutôt consécutif. Si l'on considère les résultats des jeunes Français de 15 et 16 ans en anglais en fin de collège (Ministère de l'éducation nationale, 2004), on peut interpréter leur dernière place au palmarès de sept pays comme un signe qu'en France, l'accumulation de nombreuses années d'études d'une ou de deux langues, pour diverses raisons que je n'ai pas l'espace de développer ici, ne fonctionne pas bien.

On pourrait du reste imaginer que des langues servent de tremplins à d'autres: l'espagnol au portugais, le russe au polonais, l'allemand au néerlandais. Mais quid de l'anglais? En tant que langue diachroniquement hybride (fond lexical et quelques aspects de la morphosyntaxe romanisées, mais système grammatical - verbal par ex. globalement germanique), pourquoi ne pas envisager cette langue comme un socle à l'apprentissage linguistique diversifié, y compris celui du français langue de scolarisation ${ }^{18}$

\subsubsection{Un statu quo amélioré : insérer l'anglais dans une didactique du plurilinguisme}

Ce scénario est au cœur même des débats que nombre de chercheurs ont depuis quelques temps (cf. le colloque Acedle de janvier 2008, la réunion du 14 novembre 2008 à l'IUFM de Paris à l'initiative de l'Association pour le développement de l'éducation bilingue (ADEB) et la journée NeQ 2009 organisée à Tours en janvier 2009 dont s'inspire ce texte, etc.). Si l'anglais triomphe sans que nous ne puissions y faire quoi que ce soit, alors il est temps de se dire qu'il faudra vivre avec, qu'il fera de plus en plus partie de notre identité langagière ${ }^{19}$ et que cela doit désormais devenir, malgré le paradoxe apparent, un outil de promotion de la diversité. Il s'agirait du statu quo actuel, agrémenté d'un petit plus, en quelque sorte : un apprentissage citoyen qui permettrait de comprendre qu'apprendre les langues, c'est aussi comprendre la diversité de notre entourage (voir Candelier, 2003) et encourager certaines formes de cohésion sociale (Guillaume, 2005).

37 Ces approches " pluri » (à savoir à la fois plurilingues et plurielles, cf. Candelier 2008), si l'on ne se limite pas à leurs vertus d'aide à l'apprentissage d'une ou de plusieurs langues, peuvent aussi se concevoir comme des «manières d'être» (Beacco, 2005) et des moyens d'accéder à l'apprentissage de la tolérance, de la diversité et de la pluralité (voir Coste et al., 2007 ; Coste, 2008). Intégrer l'anglais à une éducation plurilingue impose donc un dépassement de l'enseignement de la langue dans la mesure où on vise ici, outre des compétences langagières, la construction d'une citoyenneté démocratique (Breidbach, 2003). 

les Français (et les francophones en général) ont implicitement accepté le déclin du supposé universalisme français (et de la langue française). Il est temps de dire et d'enseigner que nous sommes inscrits dans l'histoire, pas une histoire d'académicien, livresque et figée, mais celle qui nous fait évoluer, avancer, communiquer avec les autres, nos voisins, avec les moyens à notre disposition. Tout un chacun aura compris que maintenant, la communication avec le voisin se fait souvent en anglais ${ }^{20}$. Le but de ce scénario est qu'elle puisse aussi se faire par d'autres langues, en plus de l'anglais.

Aussi, c'est dans ce scénario et à notre époque, dans notre société aux prises avec les mutations de la mondialisation / globalisation (voir Todd, 2008), qu'il faut désacraliser la langue (Klinkenberg, 2001) ${ }^{21}$ : déstabiliser les hiérarchies entre langues, déconstruire les représentations-obstacles (Forlot, 2009a), contribuer à affranchir les langues d'associations construites par le sens commun sur des approches ethnoculturelles essentialistes (par ex.: l'Espagne et les vacances au soleil, l'arabe et le danger du terrorisme, l'allemand et la rigueur intellectuelle, etc. Cf. entre autres Boyer, 2003). L'anglais peut ici être d'une grande utilité, dans la mesure où il montre aux apprenants, à leur niveau de maturité et de métalangage, que l'anglais s'est mélangé, qu'il a évolué, qu'il a pris à certaines langues pour redonner à d'autres, qu'il est en fait un peu à l'image des peuples contemporains (notamment ceux des pays anglo-saxons) : pluriel, pluriculturel et de constitution plurilingue.

En outre, la présence incontournable de l'anglais de nos jours dans les programmes d'enseignement, de l'école à l'université, permet une double (ou multiple) perspective : celle du français, langue de la collectivité nationale et de la scolarisation dans nos pays francophones, ainsi que celle de l'anglais, langue issue d'un apprentissage linguistique scolaire partagé par de plus en plus d'apprenants. Le cas échéant, s'y ajoutent des langues de la migration, régionales, familiales ou patrimoniales. Ainsi, procéder à des activités métalinguistiques à l'école (réfléchir aux formes et au fonctionnement de la langue du primaire au secondaire) sur la base de plusieurs idiomes, proches ou éloignés typologiquement (cf. Éloy, 2004), ne fait pas qu'élargir les horizons de la comparaison: cela permet, pédagogiquement parlant, d'en accentuer l'efficacité de la compréhension et de la mémorisation par les apprenants.

\subsection{L'avenir du plurilinguisme dans les apprentissages : la France est-elle prête?}

41 La grande question, davantage que l'anglais à l'hypercentre des apprentissages, me semble être celle de la société que nous construirons à l'avenir. Pour reprendre une partie du titre de l'introduction de Véronique Castellotti au n ${ }^{\circ} 11$ de Glottopol (2008a), la question des langues est intrinsèquement liée à celle de "la construction d'une école et d'une société plurielle". Or, à l'image de notre propre histoire linguistique, l'anglais à l'école en France obéit de nos jours davantage à ce que Skuttnab-Kangas (2000) conçoit comme un paradigme "diffusionniste", à l'opposé d'un paradigme idéal qui serait celui d'une écologie linguistique. Bien souvent d'ailleurs, les propres positionnements des défenseurs de la langue française vis-à-vis de l'équilibre entre langues ne font qu'accentuer le paradigme diffusionniste, alors que nous souhaiterions le juguler ${ }^{22}$.

42 En ce début de siècle qui sera encore plus marqué par les brassages, les mobilités et les diversités que les précédents, il est sans doute temps que nous réfléchissions, tant à 
l'école que dans les autres sphères de la société, à la place d'une diversité globale, ou, comme l'appellent certains écrivains créoles comme Constant ou Chamoiseau, la diversalité (Confiant, 2006). C'est aussi la raison pour laquelle je laissais entendre plus haut qu'un intense travail de culture sociolinguistique reste à faire à tous les niveaux, s'adressant aux enfants comme à leurs parents, aux acteurs et aux décideurs de l'école à l'université, sans oublier les Instituts de formation des maîtres (Forlot, 2006 ; 2009a ; Young, 2008a ; Young \& Mary, 2008).

\section{Conclusion : de la polémique au réalisme}

Je terminerai par quelques propos, que d'aucuns trouveront sans doute polémiques. L'anglais est à l'hypercentre des apprentissages langagiers à l'école parce que nous, éducateurs, n'avons pas encore convaincu les décideurs sur les dangers des paradigmes monolingues. Par peur de ce que l'on appelle en France le "communautarisme", on a finalement ouvert les portes à des tendances unicistes (Castellotti 2009, à par.), monolingues et dominatrices, notamment dans le domaine des langues. Le résultat est que l'on se demande pourquoi les Français sont si demandeurs d'apprentissage de l'anglais tout en étant généralement peu performants dans cette langue et peu admiratifs de ce qu'ils appellent le "modèle américain". On aurait sans doute été avisé de développer un travail sur l'arabe, le vietnamien, le portugais, le breton, l'occitan, en parallèle avec les langues plus traditionnelles du système scolaire. Cela aurait permis de travailler sur les stéréotypes, de créer une culture de l'apprentissage des langues, de la diversité, de la variation, de la tolérance. Cela permettrait sans doute d'éviter d'entendre, de la bouche de certains professeurs stagiaires, que le picard qu'ils parlent est une abomination ou encore que ce sont aux Arabes d'apprendre le français, et pas l'inverse... (cf. Forlot $2006 ; 2009 a)$.

Cependant, quelques mots de réalisme s'imposent, avant de clore cet article. Certaines prises de position faisant la promotion de la diversité et de la pluralité (voir par ex. Skuttnab-Kangas 2000) ont à mon avis une tendance exagérée à mettre la question linguistique sur le devant de la scène, de façon évidente et systématique. Mais est-il si sûr que dans l'ensemble, nos problèmes d'hégémonie, de domination, de monoculture, de globalisation... soient essentiellement linguistiques? En d'autres termes, il conviendrait que les sociolinguistes et les didacticiens s'intéressent aussi aux langues en les problématisant de façon socio-anthropologiques, c'est-à-dire en les considérant comme des éléments, parmi d'autres, constitutifs des relations de domination et d'inégalité dans les rapports sociaux et dans l'accès à tous les types de ressources (professionnelles, financières, culturelles...). Il est possible que la valorisation des attitudes "pluri" passe par l'acceptation politique que certains pays, dont la France ou la Belgique (voir Lucchini et al., 2008) sont devenus des sociétés multiculturelles.

Tout cela signifie sans doute que la diffusion de l'idéologie - au sens noble du terme du plurilinguisme devrait être accompagnée d'une vision sociétale qui pourrait en affiner la compréhension et donc contribuer à une mise en œuvre, ne serait-ce que modeste, de celui-ci. Cette option demanderait sans doute d'être approfondie de façon théorique et empirique, mais on peut émettre l'hypothèse que la constitution d'une société au fonctionnement multiculturel pourrait par exemple permettre aux principes de la didactique du plurilinguisme de ne pas rester à l'état de discussions et de débats scientifiques entre universitaires... Dans cette société perçue comme plurielle au sens 
large, la langue anglaise prendrait bien sûr une place de choix, très certainement charnière, mais dans un rapport plus égalitaire vis-à-vis des autres idiomes.

\section{BIBLIOGRAPHIE}

Alén Garabato C., Auger N., Gardies P. \& Kotul E. (2003). Les représentations interculturelles en didactique des langues-cultures. Enquêtes et analyses. Paris : l'Harmattan.

Attali, J. (sous la présidence de.) (2008). Rapport de la commission pour la libération de la croissance française. Paris : XO Éditions-La Documentation française.

Bautier, É. (1997). Pratiques langagières, pratiques sociales. De la sociolinguistique à la sociologie du langage. Paris : L'Harmattan.

Beacco, J.-C. (2000). Les dimensions culturelles des enseignements de langue. Paris : Hachette.

Beacco, J.-C. (2005). Langues et répertoires de langues : le plurilinguisme comme «manière d'être» en Europe. (Étude de référence du Guide pour l'élaboration des politiques linguistiques éducatives en Europe - De la diversité linguistique à l'éducation plurilingue.) Strasbourg: Conseil de l'Europe.

Blondin, C., Candelier, M., Edelenbos, P., Johnstone, R., Kubanek-German, A. \& Taeschner, T. (1998). Les langues étrangères dès l'école maternelle ou primaire. Conditions et Résultats. Bruxelles : De Boeck Université.

Bourdieu, P. (1982). Ce que parler veut dire : l'économie des échanges linguistiques. Paris: Fayard.

Bourdieu, P. (1984). Questions de sociologie. Paris : Les Éditions de Minuit.

Boyer, H. (2003). De l'autre côté du discours. Recherches sur les représentations communautaires. Paris : L'Harmattan.

Breidbach, Stephan. (2003). Le plurilinguisme, la citoyenneté démocratique en Europe et le rôle de l'anglais. Strasbourg : Conseil de l'Europe, Division des Politiques linguistiques.

Cameron, D. (2000). Good to Talk ? Londres: Sage.

Candelier, M. (dir.) (2003). Janua Linguarum - la porte des langues. L'introduction de l'éveil aux langues dans le curriculum. Strasbourg : Éditions du Conseil de l'Europe.

Candelier M. (2008), "Approches plurielles, didactiques du plurilinguisme : le même et l'autre". Cahiers de l'Acedle, vol. 5, n 1. pp. 65-90.

Castagne, E. (2008). "Les langues anglaise et française : amies ou ennemies ? ". Études de Linguistique Appliquée $\mathrm{n}^{\circ}$ 149. pp. 31-43.

Castellotti, V. (dir.) (2001). D'une langue à d'autres : Pratiques et représentations. Rouen, Presses Universitaires de Rouen.

Castellotti, V. (2008a). "Vers la construction d'une école et d'une société plurielles : des notions en débat, des orientations à construire", Glottopol n 11 . p.2-11. 
Castellotti, V. (2008b). "L'école française et les langues des enfants : quelle mobilisation de parcours plurilingues et pluriculturels ? ". In Chiss, J.-L. (dir.). Immigration, école et didactique du français. Paris : Didier. pp. 231-279.

Castellotti, V. (à paraître, 2009). "L'anglais, le français, le chinois et les autres : quelle(s) intégration(s) des apprentissages ? ". In Forlot, G. (Dir.) L'anglais et le plurilinguisme. Pour une didactique des contacts et des passerelles linguistiques. Paris : L'Harmattan.

Castellotti, V. \& Moore, D. (2002). Représentations sociales des langues et enseignement, Etude de référence pour le Guide pour l'élaboration des politiques linguistiques-éducatives en Europe, Strasbourg : Conseil de l'Europe.

Cenoz, J., Hufeisen, B. \& Jessner, U. (dir.) (2001). Cross-linguistic Influence in Third Language Acquisition: Psycholinguistic Perspectives. Clevedon, Multilingual Matters.

Conseil de l'Europe (2001). Cadre européen commun de référence pour les langues : Apprendre, enseigner, évaluer. Paris : Didier.

Chini, D. (2003) "Formation académique et pratique professionnelle : quel entre-deux ? ". Études de Linguistique Appliquée $\mathrm{n}^{\circ}$ 129. pp. 9-20.

Chini, D. (à paraître, 2009). "Premières rencontres avec la langue de l'autre ou les fondements d'une compétence plurilingue". In Gaspari F., Marie-Laverrou F. \& Parsons M. (dir.). Premières rencontres avec l'autre (Rives/Cahiers de l'Arc Atlantique ${ }^{\circ} 1$ ). Paris : L'Harmattan.

Commission européenne (2008). Chiffres clés de l'enseignement des langues à l'école en Europe. Agence exécutive "éducation, audiovisuel et culture" - Eurydice.

Confiant, R. (2006). "Créolité et francophonie ; un éloge de la diversalité". Disponible en ligne. http://www.potomitan.info/articles/diversalite.htm. Consulté le 10/05/2009.

Coste, D. (2001). "De plus d'une langue à d'autres encore. Penser les compétences plurilingues ? ". In Castellotti, V. (dir.). D'une langue à d'autres : Pratiques et représentations. Rouen : Presses Universitaires de Rouen. pp.191-202.

Coste, D. (2008). "Éducation plurilingue et langue de scolarisation". Cahiers de l'Acedle, vol. 5, nº 1 . pp. 91-107.

Coste, Daniel, Marisa Cavalli, Alexandru Crișan, Piet-Hein Van de Ven. 2007. Un document européen de référence pour les langues de l'éducation? Strasbourg : Conseil de l'Europe, Division des Politiques linguistiques.

Crystal, D. (1997). English as a Global Language. Cambridge: CUP

Culioli, A. (1990). Pour une linguistique de l'énonciation. Paris : Ophrys.

Eloy, J.-M. (2004). "Langues proches: que signifie de les enseigner? ". Études de Linguistique Appliquée $\mathrm{n}^{\circ}$ 136. pp. 393-402.

Fairclough, N. 2006. Language and Globalization. Oxford: Routledge.

Forlot, G. (2006). "Des pratiques aux stéréotypes sociolinguistiques d'étudiants-professeurs. Résultats préliminaires d'une enquête et pistes de recherche". Spirale $\mathrm{n}^{\circ}$ 38. pp. 123-140.

Forlot, G. (2009a). "Vers la déconstruction d'un apprentissage idéologique : Des "représentationsobstacles" dans l'enseignement des langues en France". Cahiers de l'Acedle, vol. 6, nº 1. pp. 69-91.

Forlot, G. (à paraître, 2009b). "Le rôle de l'anglais dans les apprentissages linguistiques : appropriation et capitalisation d'une culture du contact des langues". In Forlot, G. L'anglais et le plurilinguisme. Pour une didactique des contacts et des passerelles linguistiques. Paris : L'Harmattan. 
Forlot, G. (dir) (à paraître, 2009c). L'anglais et le plurilinguisme. Pour une didactique des contacts et passerelles linguistiques. Paris : L'Harmattan.

Forlot G. \& Beaucamp J. (2008). "Heurs et malheurs de la proximité linguistique dans l'enseignement de l'anglais au primaire". Études de Linguistique Appliquée nº 149. pp. 77-92.

Gaonac'h, D. (2006). L'apprentissage précoce d'une langue étrangère : le point de vue de la psycholinguistique. Paris : Hachette.

Guillaume, A. (coord.) (2005). "Les langues pour la cohésion sociale". Les Langues Modernes $n^{\circ} 4$. Paris : APLV.

Hagège, C. (2006). Combat pour le français. Au nom de la diversité des langues et des cultures. Paris : Odile Jacob.

Heller, Monica. (2002). Éléments d'une sociolinguistique critique. Paris : Didier.

Heller, M. (2007). "Bilingualism as ideology and practice". In Heller, M. (dir). Bilingualism : A Social Approach. Basingstoke: Palgrave. pp. 1-22.

Hermans, S. (1997). "Promoting foreign language competencein the European community : the Lingua programme". World Englishes, vol. 16, $\mathrm{n}^{\circ}$ 1. pp. 45-55.

Jessner, U. (2006). Linguistic Awareness in Multilinguals. English as a Third Language. Edinburgh: Edinburgh University Press.

Jucquois, G. (2003). "Hybridité". In Ferréol, G. et Jucquois, G., Dictionnaire de l'altérité et des relations interculturelles. Paris : Armand Colin. pp. 147-153.

Kachru, B. (1996). The Alchemy of English: The Spread, Functions and Models of Non-native Englishes. Oxford: Pergamon Press.

Klinkenberg, J.-M. (2001). La langue et le citoyen. Paris : PUF

Kramsch, C. (1998). Language and Culture. Oxford: Oxford University Press.

Labrie, N. (1993). La construction linguistique de la Communauté européenne. Paris : Honoré Champion.

Lucchini, S., Hambye Ph., Forlot G. \& Delcourt I. (2008). Francophones et plurilingues. Le rapport au français et au plurilinguisme des Belges issus de l'immigration, Fernelmont : Éditions Modulaires Européennes (coll. Français et Société nº 19).

Makoni S. et Pennycook, A. (dir.) (2007). Disinventing and Reconstituting Languages. Clevedon: Multilingual Matters.

Matthey, M. (dir.) (1997). Les langues et leurs images. Neuchâtel : IRDP.

Ministère de l'éducation nationale (2004). "Evaluation des compétences en anglais des élèves de 15 ans à 16 ans dans sept pays européens", publication 04.01. Paris : MEN-DEPP. Consulté le 28/12/2008 sur http://www.education.gouv.fr/stateval

Ministère de l'éducation nationale (2007). Repères et références statistiques sur les enseignements, la formation et la recherche (RERS). Paris : MEN-DEPP. Consulté le 05/12/2008 sur www.education.gouv.fr/cid21641/reperes-et-references-statistiques.html

Moore, D. (2007). Les représentations des langues et de leurs apprentissages. Références, modèles, données et méthodes. Paris : Didier.

Paveau, M.A. \& Rosier, L. (2008), La langue française : Passions et polémiques. Paris : Vuibert.

Pennycook, A. (2007). Global Englishes and transcultural Flows, Oxford: Routledge 
Phillipson, R. (2003). English-Only Europe ? Challenging Language Policy. Oxford: Routledge.

Prendergast, C. (2008). Buying into English. Language and Investment in the New Capitalist World. Pittsburgh: University of Pittsburgh Press.

Skuttnabb-Kangas, T. (2000). Linguistic Genocide in Education - or Worldwide Diversity and Human Rights? Mahwah, NJ: Lawrence Erlbaum.

Todd, E. (2008). Après la démocratie. Paris : Gallimard.

Truchot, C. (1990). L'anglais dans le monde contemporain. Paris : Le Robert.

Truchot, C. (2008). Europe : l'enjeu linguistique. Paris : La Documentation française.

Young, A. (2008a) "Diversity as an asset: Multiple language integration", in Dooly, M. \&

Eastment, D (dir.). How we're going about it. Teachers' voices on innovative approaches to teaching and learning languages. Newcastle upon Tyne: Cambridge Scholars Publishing, pp.51-65.

Young, A (2008b). "Parent-teacher partnerships co-constructing knowledge about languages and cultures in a French primary school", in Kenner, C. \& Hickey, T. (dir.), Multilingual Europe: Diversity and Learning, Stoke-on-Trent, Trentham Books.

Young, A. \& Mary, L. (2008) "Comment former les professeurs stagiaires pour une meilleure prise en compte de la diversité linguistique et culturelle à l'école ?". Le français aujourd'hui. nº 164 .

Paris : Armand Colin.

Zarate, G. 1993. Représentations de l'étranger et didactique des langues. Paris : Didier.

\section{NOTES}

1. Alors que la communication interne à l'U.E. est, quant à elle, régie de façon stricte par les principes du multilinguisme (documentation, traduction et interprétariat multilingues)

2. Du reste, les conclusions du réseau Eurydice pour 2008, se basant sur les données d'Eurostat, donnent le même type de profil pour l'éducation scolaire : $95 \%$ de l'ensemble des langues apprises à l'école se trouvent dans le groupe suivant: anglais, allemand, allemand, espagnol, russe.

3. Mais beaucoup moins souvent par les chercheurs en didactique des langues : voir Blondin et al., 1998 ; Gaonac'h, 2006 ; Chini, à par. 2009.

4. Ce qui a des implications bien entendu linguistiques, comme la référence devenue rare au "prénom" comme Christian name, mais plutôt comme first name.

5. On l'aura compris, ce qui se joue ici est surtout la rentabilité commerciale pour les éditeurs de ces manuels et de tout l'appareillage pédagogique qui les accompagne : cassettes, CD Rom, DVD, logiciels...

6. Ceci tranche nettement avec l'analyse que fait J.-C. Beacco de la tendance classique à l'indifférenciation entre langue et civilisation dans les cours de langues (et de FLE notamment) dans le secondaire et aux adultes (Beacco, 2000 : 76-78).

7. À l'instar de notre fameux "mais-ou-est-donc-or-ni-car", que chacun se souvienne de ses listes de verbes irréguliers anglais à apprendre à la fin du manuel, ou des prépositions allemandes "aus-bei-mit nach-seit-von-zu" suivis du datif en allemand, à retenir forcément avec le tableau des déterminants appropriés!

8. Même si les chiffres des tableaux 4 et 5 , avec les précautions interprétatives d'usage, mettent en relief une pratique communicative plus importante dans l'académie de Strasbourg que dans celle d'Amiens. 
9. Une approche des pratiques langagières "réelles" - faute d'un meilleur adjectif icipermettrait d'appréhender celles-ci comme ne se limitant pas aux aspects proprement linguistiques, même si la langue en demeure un volet fondamental. Bautier (1997 : 13) rappelle à juste titre que la notion de pratiques langagières met de l'intelligibilité dans la diversité et l'hétérogénéité des phénomènes liés au langage. On proposera de définir ici les pratiques langagières comme des activités conscientes (ou réflexives) et inconscientes mettant en jeu une interaction de phénomènes liés au langage (pris dans sa globalité), activités dont l'exécution, située dans le temps et l'espace, a systématiquement pour origine la situation de l'acteur dans une structure sociale composée d'espaces discursifs et pour effet de le re-situer dans cette structure.

10. Voir Chini, 2003, pour une intéressante perspective de l'épreuve pré-professionnelle du CAPES.

11. J'utilise ce terme pour dériver le mot "marchandise", à l'instar de l'anglais "commodification", qui sert à analyser la langue comme un bien (commodity, en anglais) capitalisable dans diverses situations (voir notamment Cameron, 2000 ; Heller, 2002).

12. Il faut lire Alastair Pennycook notamment pour comprendre les simplifications opérées par le modèle des "World Englishes" à la Kachru (1996) et Crystal (1997) ou, au contraire, le modèle, inversement caricatural selon lui, de l'impérialisme linguistique de Phillipson (2003), SkuttnabbKangas (2000), etc. Pennycook penche plutôt pour le concept de "Global Englishes", parlers se diffusant à travers le monde via la mondialisation, mais se trouvant réappropriés localement sans que cela ne pose de difficultés de nature sociolinguistique et sans que cela ne soit à proprement parler de l'anglais, construction simplificatrice, selon lui.

13. On remarque depuis la rentrée de 2008 un retour, excessif selon moi, de ce que l'on appelle pudiquement la "trace écrite", retour sans doute lié aux orientations philosophiques des nouveaux programmes français de l'enseignement primaire, adossés au "socle commun de connaissances et de compétences" mis au point en 2006.

14. Dans une perspective de sociolinguistique "sociale" qui est la mienne, les adjectifs "épilinguistique" et "métalinguistique" ne renvoient pas tout à fait aux définitions classiques (notamment à celle de Culioli 1990). J'emploie le premier pour faire référence aux positionnements vis-à-vis des statuts des langues, de leurs valeurs sur un marché, ou de leur évaluation selon des critères esthétiques, par exemple, ou encore par rapport à des discours s'inscrivant dans une vision essentialiste associant langues et conduites ethno-identitaires. L'adjectif "métalinguistique" fait en revanche ici référence aux positionnements et aux discours sur la langue elle-même, dans ses aspects formels et structurels (par ex. les déclinaisons, temps et aspects, l'orthographe, etc.). Cf. Forlot, 2009a.

15. J'utilise l'adjectif "prétendu" parce qu'à l'instar de R. Phillipson (2003), je ne pense pas que l'anglais soit véritablement une lingua franca, dans la mesure où (a) cette langue est parlée par de nombreux locuteurs en L1 et en LS qui sont de facto en situation de domination linguistique lorsqu'il s'agit de chercher des bourses d'études ou de l'emploi requérant l'usage de l'anglais (cf. les fréquentes petites annonces recherchant des natifs de l'anglais); (b) deux formes d'anglais triomphent actuellement (anglais d'Angleterre et des États-Unis) au détriment des autres formes d'anglais L1 et LS.

16. Deux illustrations, mais elles sont légion : dans une petite ville du Nord, à la frontière francobelge (Flandre), les parents ont demandé aux autorités compétentes de généraliser l'enseignement de l'anglais dans certaines écoles où persistait une relative diversité de choix, ce qui s'est fait naturellement au détriment du néerlandais enseigné dans le primaire. Dans une école de l'Oise, les parents ont exigé le remplacement de l'enseignement de l'allemand dans une école au profit de l'anglais, au nom du principe de continuité école-collège.

17. Si j'en avais l'espace, j'insisterais sur l'importance de ces "discours circulants" dans la structuration de l'espace social éducatif. Ces discours circulant par différents biais (médiatiques 
notamment, mais pas seulement) construisent et reproduisent les hiérarchisations et les dynamiques de dominations et de minorations entre les acteurs et les objets sociaux (voir Heller, 2002).

18. Je renvoie ici aux nombreuses publications en anglais sur la question (cf. Cenoz et al., 2001), à quelques écrits précurseurs sur la question en français (cf. Coste, 2001), ainsi qu'au récent numéro 149 des Études de Linguistique Appliquée (cf. notamment Castagne, 2008; Forlot \& Beaucamp, 2008, sur la nécessité de développer une approche néo-contrastive comme volet métalinguistique de l'apprentissage des langues). Cf aussi Forlot, 2009c, à paraître.

19. Ne serait-ce que par les nombreux contacts que nos langues ont avec lui : pensons aux SMS, à Outlook, à MSN, au bluetooth, au hard discount...

20. Une courte anecdote en guise d'illustration, ici : à la veille du colloque de l'Acedle de janvier 2008, me promenant devant la cathédrale de Strasbourg, je suis le témoin d'une rencontre entre deux groupes de lycéens, l'un d'Italie, l'autre d'Espagne. Un des Espagnols s'exclame, en souriant au groupe de jeunes Italiens : "Italia? Eh, Spanish, Italian, it's almost the same !".

21. Sans sombrer pour autant dans un relativisme outrancier vis-à-vis de la norme, dont Bourdieu (1982) a bien montré qu'il était tout aussi créateur d'inégalité sociale et de discrimination que son inverse, la fétichisation de la langue.

22. Voir la critique de Pennycook (2007 : 19-20) à l'endroit des positions d'Hagège (2006), qui selon lui ne rompent pas avec la tradition de défense d'une langue dominante, qui serait en l'occurrence le français plutôt que l'anglais.

\section{RÉSUMÉS}

Cet article brosse un portrait de la place de l'anglais dans le système éducatif français et s'intéresse tant aux raisons de la domination de l'anglais qu'à certaines approches pédagogiques parfois paradoxales qui tendent à renforcer le statut de cette langue et les pratiques monolingues à l'école. Sans négliger une réalité sociolinguistique qui fait de l'anglais la langue incontournable de nos sociétés contemporaines et mondialisées, on propose ici une réflexion sur un scénario qui permettrait à la langue anglaise de s'insérer dans une politique de la diversité et de la pluralité linguistique au sein du système éducatif.

This article examines the place of English in the French education system and addresses both the reasons for the domination of English and some paradoxical teaching practices reinforcing both the status of this language, as well as monolingual tendencies at school. While taking full account of the sociolinguistic fact that English has become the unavoidable language of our contemporary, globalized societies, what is proposed here is to ponder a scenario which would allow English to be part of an educational and linguistic policy of diversity and pluralism.

\section{INDEX}

Mots-clés : anglais, système éducatif, domination linguistique, pluralité

Keywords : english, education system, language domination, pluralism 


\section{AUTEUR}

\section{GILLES FORLOT}

Gilles Forlot est maître de conférences à l'Université de Picardie Jules-Verne (IUFM-UPJV) et membre du Laboratoire d'études sociolinguistiques sur les contacts de langues et la politique linguistique (LESCLaP, JE 2466) de cette université. Il a été chercheur à l'Ontario Institute for Studies in Education de l'Université de Toronto de 1993 à 1997 et à l'Université catholique de Louvain de 2002 à 2007.

Courriel : gilles.forlot[at]u-picardie.fr

Toile : http://www.u-picardie.fr/LESCLaP

Adresse : Centre IUFM/UPJV, 3 rue Bossuet, 60000 Beauvais, France. 